\title{
Creation of Robust Schedule for Profit Based Cooperation
}

\author{
Jiří Hodík \\ Gerstner Laboratory - Agent Technology Center, Faculty of Electrical Engineering, \\ Czech Technical University in Prague, Technická 2, Praha 6, Czech Republic \\ hodik@labe.felk.cvut.cz
}

\begin{abstract}
Method for robust schedule preparation for domain of profit based cooperation is introduced in this paper. The method aims schedules, which are prepared and concluded step-by-step during the team formation phase. The method takes into account domain specifics, like incomplete information about the partners, and their various reputation and skill levels. The method is based on critical path dispatching rule, which is widely used in heuristics for $n p$ hard and continuous scheduling problems. Experimental results are presented for various workflow structures and other configurations of the domain.
\end{abstract}

\section{Introduction}

In the domain of profit based cooperation, the cooperators are motivated to join (and to stay in) the team (e.g. Supply Chain and some types of Virtual Organizations) by expected profit. We concentrate to teams with one leader subcontracting the other partners mutually independently. Sometimes the leader has to conclude contracts with the others without having prepared whole schedule; it is to be prepared online during the negotiation about involvement the partners. Contrary to the intra-organizational scheduling, this scheduling adds requirements and constraints to scheduling methodologies. Together with complexity of the scheduling problem it is too hard to prepare valuable estimation of schedule until it is negotiated with the partners. Moreover, the leader has limited access to information about the partners' resources and such information may be distorted either by intention of partners to improve impression about their competencies, or by inexperienced guess of them.

When any already concluded due-date is not kept and if there is not enough slack, the processing of consequent tasks is delayed. It is critical mainly when it is not possible to reschedule the consequent tasks either because of high utilization of facility, or because of high penalties for not keeping the concluded due-dates.

The targeted domain relates to the supply chain planning and scheduling models class, which includes medium planning models mainly on an entire network level (e.g., project planning and scheduling or lot sizing models), as well as detailed scheduling models mainly on work-centers levels (e.g., job shop scheduling). The features crucial for the top level schedule creation are: 
- Members. They may be autonomous, self-oriented and distributed.

- Limited access to information. Nobody has direct access to the partners' internal information, neither the team leader.

- Partners with limited reliability. Presumption about partners' trustworthiness may be helpful in negotiation about resources allocation.

- Concluded contracts. Withdrawing from a contract or changing it is limited by the concluded rules and the affected partner's willingness.

There are several scenarios for team creation phase; all of them provide the plan and schedule for further team operations. The schedule is pre-negotiated with the partners before its conclusion, or involvements of the partners are being concluded continuously during the scheduling process. Reasons for continuous concluding are: (i) partners provide an expected completion time of the task but they do not share knowledge about available resources; and (ii) partners' resources may be shared within the partner; once dedicated for a task they cannot be used for the other one.

The scheduling algorithm is interlinked with a protocol for negotiation about tasks processing commitments. Often, it is possible to pre-negotiate involvement of the members, or mechanisms for its update according to current state of the team exist. There are several works extending basic negotiation protocols by non-bounding offers and commitments to improve quality of the consortium and its schedule like LAP [5], C-CNP [7], RC-CNP [1], and RBVO formation protocol [8]. Although any such mechanism for involvement modification may be implemented, there is a condition of available resources. Without resources, the schedule update is excluded although the negotiation protocols would support the updating process.

\section{Scheduling Methods}

Many scheduling methods for (not only hard) scheduling problems are based on priority rules comparing tasks priorities from attributes of tasks, machines, and actual state of the world. The rules are static, or dynamic (time is important as well). There are several schedule measures and objectives. Example of a schedule objective is the makespan, which is defined as the latest completion times of mutually related tasks.

\subsection{Critical Path Method}

The Critical Path Method (CPM) is a common method for scheduling set of tasks with defined precedence constraints. The objective to be optimized is the makespan. The method concentrates to finding and optimization of critical path under these constraints: (i) the processing time of a task is fixed, (ii) no other resource than machine time is required, and (iii) there are enough of parallel machines. The CPM inspired for Critical Path dispatching rule, which employs precedence constraints and processing times of tasks. From them the strings of processing times are counted and the head of the longest one is given the highest priority. 


\subsection{Robustness of Schedule}

The robust schedule is required when any rescheduling may negatively influence the schedule objectives (e.g., too expensive, late, or no alternate resources). The robust schedule has "the ability to satisfy performance requirements predictably in an uncertain environment" (Pape in [4] in [2] by Davenport et al.). Pinedo presents four examples how to create a more robust schedule [6]:

- Inserting idle/slack times. Depending on a rule, the idle times may be equal or depending on size/priority of foregoing task.

- Scheduling less flexible jobs/tasks first. Each task may be influenced by aggregated perturbations of its predecessors, for the less flexible ones this risk should be minimized.

- Postponing the processing of an operation only if really needed. Contrary to the inventory cost point of view it is better to start as soon as possible.

- Bottleneck machine utilization optimization. As the bottleneck has high impact on

- facility capacity and makespan, is should not wait for a task.

We suggest extending the Pinedo's list of potentials to increase the robustness by:

- Reliable partners. The ones with higher probability to keep concluded dates should be preferred.

Various method for computation of idle times is explored e.g. by Davenport et al., who concentrate to using robustness technique for scheduling of job-shops with totally ordered tasks in domain with full access to control over the machines [2].

To evaluate robustness of the schedule, the measure must be defined. The robustness may be counted from the schedule as it is prepared, or evaluated after the scheduled tasks (real or simulated) execution. Pinedo defines a concept, which evaluates robustness of the original schedule. The measure is based on the amount of weighted slacks between the tasks' completion times and due-dates [6]. Kouvelis and $\mathrm{Yu}$ suggest criteria to evaluated schedule robustness from simulated performance [3]:

- Absolute robustness. The worst scenario performance is the key.

- Robust deviation. Each scenario performance is evaluated against the optimal one to get the deviation. The worst deviation got is the key.

- Relative robustness. Similar to the robust deviation but the percentage deviation from the optimal case for the scenario is used.

\section{Scheduling Process}

The presented scheduling algorithm is based on CPM. The makespan and schedule robustness are the optimized objectives. The idea is that the critical tasks should be preferred during scheduling. Contrary, as the expected processing times are known after their negotiation, they may differ from the nominal ones. Therefore the critical 
path is not known until the whole schedule is prepared. It is being estimated and modified during the scheduling process. Simultaneously, keeping the negotiated times is not guarantied and therefore there is a request for robust schedules to be able to process whole job of the team.

\subsection{Formal Description of the Scheduling Problem}

From the task definition a nominal processing time is known. Actually, due to various capabilities and available resources, the processing times negotiated with the machines ${ }^{1}$ may differ. While the capabilities are supposed to be constant during the lifecycle of the VO, the resources may vary in time.

The nominal processing time of the $\operatorname{task}^{2} j$ is $p_{j}$. For starting time $S_{j}$, the nominal completion time is $C_{j}=S_{j}+p_{j}$. For processing of task $j$ on machine $i$ the concluded completion and processing times are $C_{i j}$ and $p_{i j}$. There may be also an expected completion time $C^{\prime}{ }_{i j}$, which is delayed by $\delta_{i j}$. The expected processing time is $p_{i j}^{\prime}=C_{i j}^{\prime}\left(\delta_{i j}\right)-S_{i j}$; relation $p_{i j}^{\prime} \geq p_{i j}$ may be expected.

For estimation of the delay, only following is available: the task and its complexity (represented by $p_{j}$ ), concluded processing time $p_{i j}$, and confidence in machine (given as some reputation model $R_{i}$ ). The expected delay for the task $j$ is $\delta_{i j}=f\left(p_{j}, p_{i j}, R_{i}\right)$. As the concluded processing time $p_{i j}$ is counted from the machine's available resources, $p_{j}$ may be omitted and therefore $\delta_{i j}=f\left(p_{i j}, R_{i}\right)$.

\subsection{Algorithm Overview}

Estimation of the critical path in any scheduling step is based on already concluded tasks processing (on the expected completion times), and nominal processing times (for scheduled yet part of the schedule). The following is defined:

- $J O B$ is a list of all tasks, $J O B=\left\{t_{1}, \ldots, t_{n}\right\}$, where $t_{j}$ for $(1 \leq j \leq n)$ is a task from the list, and $n$ is number of tasks in the list. For any couple of tasks the finish-tostart transitive precedence constraint $t_{1} \prec t_{2}$ may be defined. The precedence graph must be acyclic.

- $\quad C L O S E$ is a list of already scheduled tasks. For every $t_{k}$ its starting time, and concluded and (if applied) expected completion times are known.

- $\quad O P E N$ is a list of not yet scheduled tasks. Let $O P E N^{\prime}$ denote a subset of tasks from $O P E N$ having their precedential tasks scheduled.

For $\forall t_{k} \in O P E N$ the earliest possible starting time is denoted by the latest completion time from set of completion times of all predecessors: $S^{\prime}{ }_{j} \geq \max \left\{C_{k}: t_{k} \prec t_{j}\right\}$. If

\footnotetext{
${ }^{1}$ To be consistent with common terminology, the work-centers of the members are called machines.

${ }^{2}$ In the definitions $j, k$ are indexes of tasks, and $h, i$ indexes of responsible machines.
} 
$\forall t_{k}\left(t_{k} \prec t_{j} \rightarrow t_{k} \in C L O S E\right)$ then $t_{j}$ may be scheduled with starting time $S^{\prime}{ }_{j}$, otherwise $S^{\prime}{ }_{j}$ denotes that final $S_{i j} \geq S^{\prime}{ }_{j}$.

There are various priority rules for selection of the next task to be scheduled $\left(t_{N E X T}\right)$ from the OPEN' list. We have adopted following ones:

- Any ready task rule, by that any task ready to be scheduled is chosen.

- Nominal critical path rule, the next task is chosen according to the critical path counted from nominal processing times.

- Current critical path rule, the next task is chosen according to the critical path, which is updated according already existing schedule fragments.

- Longest chain of successor rules, the next task is chosen as the head of the longest chain of not scheduled tasks yet.

When the $t_{\text {NEXT }}$ is defined, the negotiation about its assignment and completion time is performed. We consider basic negotiation protocol which for defined starting time provides a set of completion times proposed by the interested machines. The decision process for determination of the winning proposal takes into account the proposed completion time and (if applied) reputation model of the proposer. We have adopted two rules for winning offer determination:

- The basic rule for offer determination: $C_{\min }\left(\forall C_{h j}\right)$, where $C_{h j}$ is by machine $h$ proposed completion time for task $j$.

- The reputation rule for offer determination: $C_{\min }^{\prime}\left(\forall C^{\prime}{ }_{h j}\right)$, where $C^{\prime}{ }_{h j}$ is the expected completion times counted from reputation models of the proposers $\left(C_{h j}^{\prime} \geq C_{h j}\right)$.

Next step to increase the schedule robustness is adding the idle time to the concluded completion time. The idle time idle ${ }_{j}$ of the task $j$ postpones earliest time for starting consequent tasks $\left(S_{k}-C_{i j}^{\prime} \geq i d l e_{j} ; t_{j} \prec t_{k}\right)$. In this work, the rules for idle time determination do not consider whether the task is the earlier one or the latter one (such information could be also beneficial as described in [2]). Our rules are:

- $\quad$ No idle time rule, idle $_{j}=0$.

- $\quad$ The nominal processing time rule based on requested robustness and nominal processing time, idle $_{j}=f\left(p_{j}\right)$.

- The concluded processing time rule is based on requested robustness and concluded processing time of the task, $i d l e_{j}=f\left(p_{i j}\right)$.

- The expected processing time rule is based on requested robustness and expected processing time of the task, idle $_{j}=f\left(p_{i j}^{\prime}\right)$. 
Algorithm 1. Process of Robust Schedule Preparation

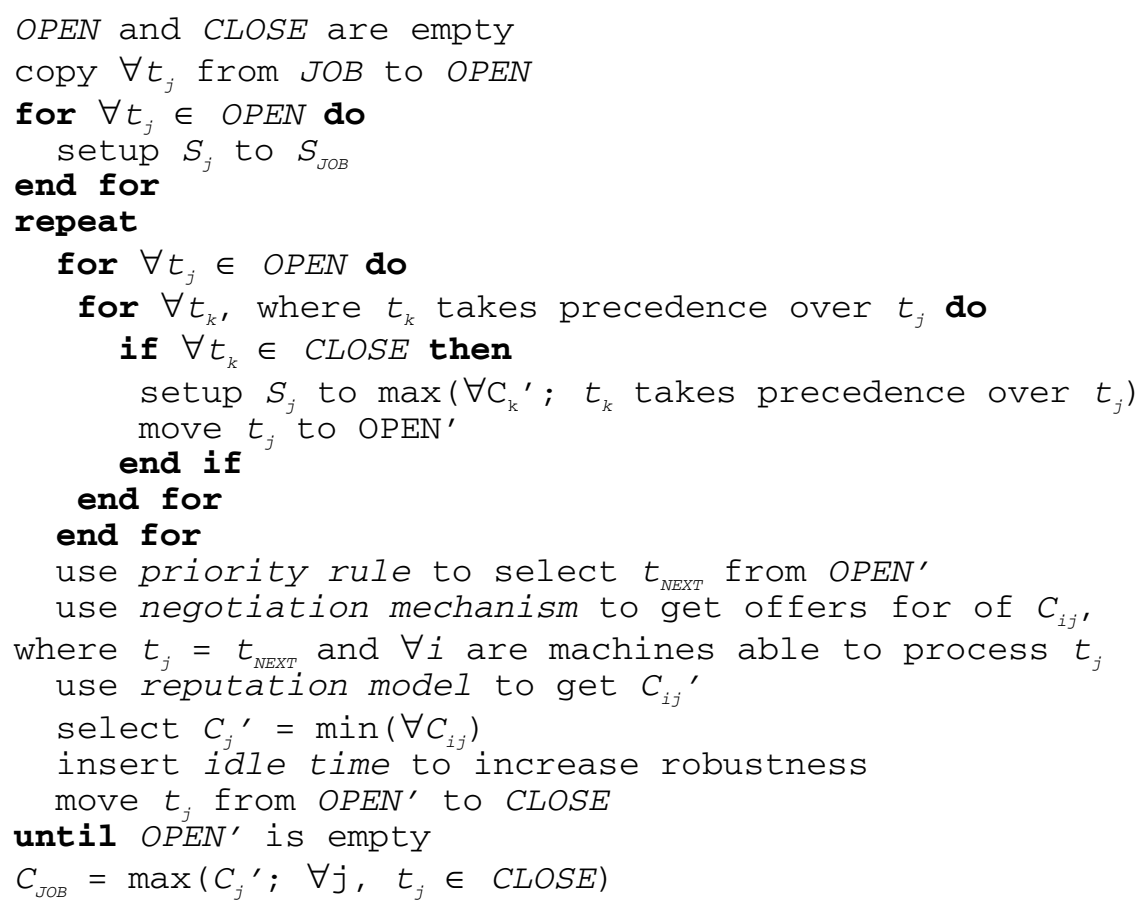

Formal description of process of preparation robust schedule preparation is presented by Algorithm 1. In the algorithm, the $S_{J O B}$ is the starting time of whole operation, the $C_{J O B}$ is its completion time.

\section{Experiments}

Several configurations of the algorithm were used; each configuration was run 20 times. In all of them simple CNP is used as a negotiation mechanism and 10 machines with limited resources shared across all tasks is involved, their capabilities vary from $70 \%$ to $90 \%$. Each machine is able to process any task. Requested robustness is $5 \%$. Number of tasks is counted as square of numbers from 1 to 7 . For each task, its resources requirements are equal to 10 times the number of tasks IDs. Three different workflow structures (see Figure 1) defined by precedence constraints are used:

- Single string, all the tasks are in one string, which is a critical path. All priority rules provide the same $t_{N E X T}$.

- All tasks in parallel, each parallel string consists of one task. The critical path consists of the longest task(s). "Any task" priority rule provides random $t_{N E X T}$, the other rules provide the same one.

- Parallel strings, all parallel strings consist of same number of tasks and have the same starting date. The tasks are distributed by rule that the following task is added to the tail of the next string. Each of priority rule may provide different $t_{N E X T}$. 


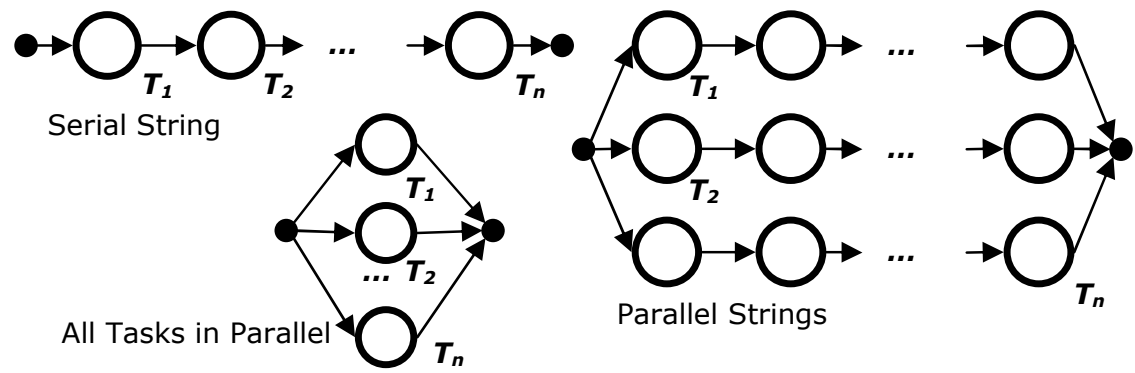

Fig. 1. Applied workflow structures

\subsection{Results}

Example of simulation runs is presented on Figure 2. The set of graphs presents makespan, robustness and scheduling order for number of tasks from 1 to 49 for parallel strings workflow structure and the priority rule of "Any task". The reputation is not assumed; no extra idle times are added.

In most of the results (not just this one), there is a break in results at position between $9\left(=3^{2}\right)$ and $16\left(=4^{2}\right)$ tasks. It is due to number of tasks that is higher than number of machines; the machines may be overloaded by already scheduled tasks.

The Table 1 presents selected results of simulations for 9 (maximum number of tasks, which is less then number of machines) and 49 tasks (maximum number of tasks in experiments, machines are overloaded). In all simulations the requested robustness was kept. The slack reserves were mainly in parallel structure on noncritical tasks if the priority rule was "Any task". For the linear-parallel structure, there is space for post-processing negotiation with partners about redistribution of slacks on the end of non-critical chains among their tasks. Also it is clear that assuming any information about the future tasks is better than scheduling any task of the ready ones. The average makespans does not differ significantly although the order of tasks

Table 1. Selected simulation results

\begin{tabular}{|l|l|l|l|l|l|}
\hline \multicolumn{2}{|c|}{} & \multicolumn{4}{|l|}{ Scheduled makespan (No. of tasks): } \\
\hline $\begin{array}{l}\text { Average robustness based } \\
\text { on processing time: }\end{array}$ & $\begin{array}{l}\text { 9 } \\
\text { (more machines than tasks) }\end{array}$ & $\begin{array}{l}\mathbf{4 9} \\
\text { (more tasks than machines) }\end{array}$ \\
\hline Structure: & Priority rule: & Nominal & Concluded & Nominal & Concluded \\
\hline Linear & Any task & 1.12 & 1.18 & 1.11 & 1.17 \\
\hline Parallel & Any task & 1.21 & 1.25 & 11.89 & 12.68 \\
\hline & CP nominal & 1.11 & 1.56 & 14.74 & 16.13 \\
\hline $\begin{array}{l}\text { Linear- } \\
\text { parallel }\end{array}$ & Any task & 1.12 & 1.17 & 1.21 & 1.28 \\
\hline & CP nominal & 1.12 & 1.17 & 1.11 & 1.17 \\
\hline & CP current & 1.12 & 1.17 & 1.11 & 1.17 \\
\hline & $\begin{array}{l}\text { Longest } \\
\text { successors }\end{array}$ & 1.12 & 1.17 & 1.12 & 1.17 \\
\hline
\end{tabular}



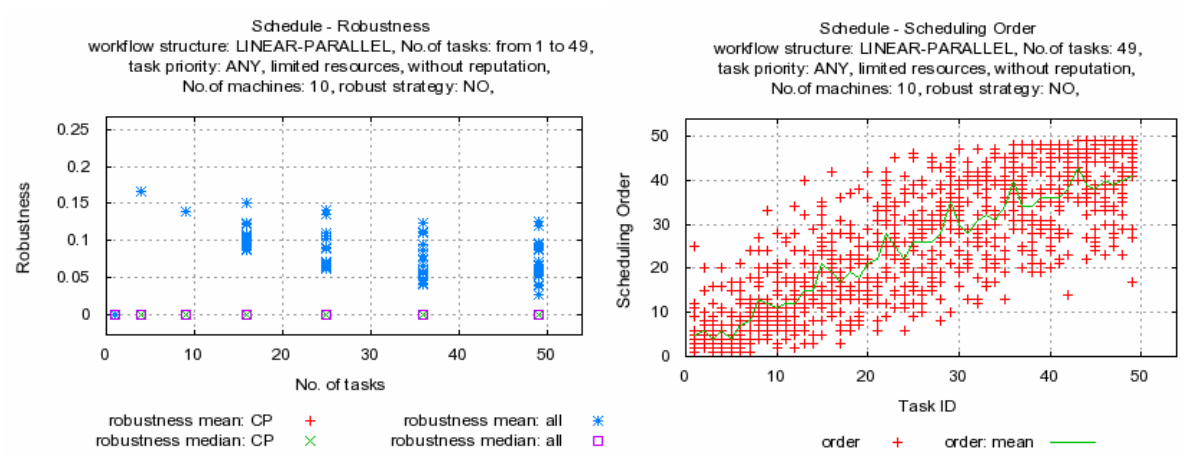

Fig. 2. Example of simulation results

scheduling varies, the main difference is in variances of robustness mainly when the reputation models are applied (not in scope of this paper).

\section{References}

1. Bíba, J., Vokřínek, J., Hodík, J.: Renegotiable competitive contract net protocol. Technical Report GL 194/08, ATG, Gerstner Laboratory, CTU (2008)

2. Davenport, A.J., Gefflot, C., Beck, J.C.: Slack-based techniques for robust schedules. In: Proc. of the Sixth European Conf. on Planning, ECP 2001 (2001)

3. Kouvelis, P., Yu, G.: Robust Discrete Optimization and Its Applications. Springer, Heidelberg (1996)

4. Pape, C.L.: Constraint propagation in planning and scheduling. Technical report, Stanford University, Palo Alto, California (1991)

5. Perugini, D., Jarvis, D., Reschke, S., Gossink, D.: Distributed deliberative planning with partial observability: Heuristic approaches. In: Proc. of the Int. Conf. on Integration of Knowledge Intensive Multi-Agent Systems. KIMAS 2007, pp. 407-412 (2007)

6. Pinedo, M.L.: Planning and Scheduling in Manufacturing and Services. Series in Operations Research and Financial Engineering. Springer, Heidelberg (2006)

7. Vokřínek, J., Bíba, J., Hodík, J., Vybíhal, J., Pěchouček, M.: Competitive contract net protocol. In: van Leeuwen, J., Italiano, G.F., van der Hoek, W., Meinel, C., Sack, H., Plášil, F. (eds.) SOFSEM 2007. LNCS, vol. 4362, pp. 656-668. Springer, Heidelberg (2007)

8. Vokřínek, J., Bíba, J., Hodík, J., Vybíhal, J., Volf, P.: RBVO formation protocol. In: 2007 IEEE/WIC/ACM International Conference on Intelligent Agent Technology (IAT 2007), pp. 454-457 (2007b) 